\title{
El papel del entrenador en la enseñanza de competencias de vida a jóvenes brasileños en riesgo de vulnerabilidad social*
}

\author{
The role of the coach in teaching of life competences at \\ risk of social vulnerability to young Brazilians
}

Recibido: 13 de marzo de 2015 | Aceptado: 21 de septiembre de 2015

\section{MAURÍCIO MARQUES ** \\ CATARINA SOUSA \\ JAUME CRUZ \\ Universitat Autònoma de Barcelona, España}

SILVIA KOLLER ***

Universidade Federal do Rio Grande do Sul, Brasil
doi:10.11144/Javeriana.upsy15-1.peec

Marques, M., Sousa, C., Cruz, J., Koller, S. (2016). El papel del entrenador en la enseñanza de competencias de vida a jóvenes brasileños en riesgo de vulnerabilidad social. Universitas Psychologica, 15(1), 129-140. http://dx.doi.org/10.11144/Javeriana. upsy15-1.peec

\footnotetext{
Artículo de reflexión. No tuvo financiación por parte de alguna entidad.

** Correo electrónico: mauriciopmarques@gmail.com; desousa.cat@gmail.com; jaume.cruz@uab.cat

***** Correo electrónico: silvia.koller@gmail.com
}

\begin{abstract}
RES UMEN
Este estudio busca investigar y analizar cómo piensan y actuan entrenadores de proyectos sociales brasileños con respecto a la enseñanza de competencias de vida a jóvenes que viven en áreas de riesgo de exclusión social. Entrevistamos a seis entrenadores de fútbol y otros deportes de distintas clases de proyectos de educación por el deporte. Se implementó un análisis cualitativo identificando y organizando meaning units, que resultó en la creación de cuatro grandes dimensiones: Trabajo en Equipo, Disciplina, Actuación y Vínculo, y Educación Integral; formadas por 28 categorías que reflejan las filosofías y prácticas de los educadores. Los resultados indican que todos los entrenadores entrevistados tienen un perfil análogo al modelo de entrenamiento de competencias de vida de Gould, Collins, Lauer y Chung (2007) que utilizan estrategias para la enseñanza de competencias de vida, como por ejemplo fomentar la autonomía, estimular el esfuerzo y dar feedback, aunque carecen de un planteamiento sistemático y tienen recursos precarios para llevar a cabo dicho modelo.

Palabras clave

competencias de vida; desarrollo positivo de jóvenes; psicología del deporte; riesgo de vulnerabilidad

\section{A B S T R A C T}

This study aims to investigate and analyze how coaches of Brazilian social projects think and act when teaching life skills to young people who live in areas of social risk. We interviewed six coaches of both football and other sports about different types of educational projects delivered through sport education. A qualitative analysis was developed through the identification of meaning units resulting in the creation of four large dimensions: Teamwork, Discipline, Performance and Bonding, and Integral Education, composing of 28 categories which reflect the philosophies and practices of these educators. The results show that all coaches that participated in this study have a similar profile "coaching life skills model" Gould, Collins, Lauer and Chung (2007) using strategies for teaching life skills, such as fostering autonomy, stimulate the effort and give feedback, but lack a systematic approach and has weak resources to carry out this model.

Keywords

life skills; positive youth development; sport psychology; at-risk
\end{abstract}




\section{Introducción}

La enseñanza a jóvenes ha ganado espacio dentro de la psicología del deporte en la última década, generando estudios y compartiendo publicaciones con temas como el entrenamiento mental para el rendimiento, que ha caracterizado el área desde su creación. Desde el punto de vista social, el deporte es más importante para la juventud como herramienta educacional para promover competencias de vida que para formar a unos pocos deportistas de élite u obtener éxitos olímpicos (Danish \& Nellen, 1997). De hecho la mayoría de niños y niñas que acuden a equipos deportivos no llegan al profesionalismo cuando son adultos.

El desarrollo positivo en jóvenes (PYD del inglés Positive Youth Development) se refiere a las formas como los niños y adolescentes pueden acumular experiencias de desarrollo óptimas a través de su participación en actividades organizadas, como el deporte (Holt \& Neely, 2011). Fundamentado en la psicologia positiva, el PYD se basa en la creencia que toda la juventud tiene potencial para vivir productivamente, establecer relaciones sociales saludables, adquirir competencias para la vida adulta, y evitar conductas de riesgo (Falcão, Bloom, \& Gilbert, 2012). Así que engloba diferentes enfoques conceptuales como las 5 C's de Richard Lerner (e.g. competence, confidence, character, caring/compasion y connection), el Athlete Development Approach, de Côté, Salmela, Trudel, Baria, \& Russell, (1995) y las competencias de vida, de Steven Danish, que estructuran programas en distintos contextos como, por ejemplo, el comunitario, el escolar o el deportivo, como los cita la revisión de Falcão et al. (2012).

En la aplicación de cualquiera de esos tres marcos en el deporte, el entrenador tiene el rol principal para la instauración y organización del ambiente para el desarrollo positivo de jóvenes (Falcão et al., 2012). Aun hoy en día, los programas de formación para ayudar a los entrenadores en la tarea educativa son escasos y, en general, se emplean en países más desarrollados, por ejemplo, el Mastery Approach to Coaching (MAC) en Estados Unidos (Smith, Smoll, \& Cummings, 2007) y el National Coach Certification Program en Canadá y el Programa de
Asesoramiento Personalizado a Entrenadores (PAPE) en España (Sousa, Cruz, Torregrosa, Vilches, \& Viladrich, 2006). Aún sin la formación adecuada, los entrenadores se sienten responsables de la educación integral de los jóvenes deportistas, como subraya el estudio de Vella, Oades y Crowe (2011). En este estudio los instructores relataron enseñar competencias específicas para el contexto deportivo y también para el contexto social y/o personal.

El concepto de competencias de vida aborda las competencias que pueden ser enseñadas en un ambiente, como el deporte, y que facilitan el éxito en otras áreas de la vida, como la escuela, o el trabajo. Estas competencias pueden ser conductuales, cognitivas, interpersonales e intrapersonales, como: comunicación positiva, establecimiento de metas, control emocional y trabajo duro (Gould, Chung, Smith, \& White, 2006). Al observar la eficacia del deporte como medio para la enseñanza de competencias de vida, el Life Skills Center coordinado por Steven Danish, creó el Programa SUPER — Sports United to Promote Education and Recreation - (Danish, Nellen, \& Owens, 1996).

En este programa SUPER participan estudiantes-deportistas de educación secundaria, con el objetivo de enseñar a otros estudiantes más jóvenes que: (a) hay modelos de estudiante-deportista efectivos y asequibles; (b) las competencias físicas y mentales son importantes tanto en el deporte como en la vida; (c) es importante el establecimiento y la consecución de metas en el deporte y en la vida; (d) se pueden superar los obstáculos que surgen en ese camino (Danish \& Nellen, 1997).

En la misma línea de trabajo, Gould y Carson (2008) han realizado una importante revisión sobre investigaciones y programas que tratan de la enseñanza de competencias de vida mediante el deporte. Incluso, proponen un modelo heurístico para la comprensión de este proceso de entrenamiento y las necesidades de investigaciones futuras. Los autores subrayan la importancia de comprender qué características y estrategias tienen los entrenadores reconocidos por el éxito en la educación de sus deportistas, como, por ejemplo, han estudiado Gould, Collins, Lauer y Chung (2007) en una investigación con entrenadores escolares destacados de 
los Estados Unidos. En este estudio se concluye que los entrenadores: (a) aunque estuvieran motivados para ganar, ellos tenían filosofías de entrenamiento que conceden gran importancia al desarrollo de competencias de vida en sus jugadores; (b) tenían la capacidad para formar relaciones sólidas y crear vínculos con los jóvenes; (c) informaron de una serie de estrategias consistentes para la enseñanza de las competencias de vida que se consideran más importantes; y (d) reconocieron que factores como el nivel socioeconómico y características familiares influyen fuertemente en el desarrollo de competencias de vida, y que los tenían en cuenta.

Marques, Sousa y Cruz (2013), hallaron resultados similares en una investigación reciente con entrenadores de proyectos sociales en España, basada en la investigación y en el guión de entrevista creado por Gould et al. (2007). Los entrenadores participantes relataron trabajar con competencias de vida que fomentan el sentimiento de competencia personal de los jóvenes, las buenas relaciones entre ellos, y la educación integral (e.g., civismo, deportividad, higiene, etc.). Las estrategias utilizadas para lograr sus objetivos mezclan el estilo de comunicación del entrenador y su actuación en la gestión de entrenamientos y partidos (Marques, Nonohay, Koller, Gauer, \& Cruz, 2015) e incluyen también en sus objetivos la participación saludable de todos y el aprendizaje que va más allá del deporte, contemplando estudios, diversión y la vida en sociedad.

Finalmente Camiré, Trudel, y Forneris (2012) utilizaron el modelo de Gould y Carson (2008) para entrevistar a entrenadores y estudiantes-deportistas canadienses, con el intento de conocer ambas perspectivas sobre las filosofías y sus estrategias para la enseñanza, además de cómo se da la transferencia de esas competencias. Las entrevistas revelaron que los entrenadores tenían en cuenta las características previas de los jóvenes y basaban sus filosofías en el desarrollo personal de ellos. Los resultados subrayaron la necesidad de la creación de vínculos entre ambos, lo cual supone por parte del entrenador tener filosofías claras y adaptadas a la realidad de cada equipo, además de utilizar sus estrategias de enseñanza con intencionalidad y de modo sis- temático. Además, tanto entrenadores como estudiantes creen que las competencias aprendidas en el deporte pueden transferirse a otras áreas de la vida.

En resumen, las investigaciones realizadas con entrenadores sobre la enseñanza de competencias de vida a través del deporte, están de acuerdo en que las competencias psicológicas solamente son asimiladas tras demostración, modelado y práctica (Gould \& Carson, 2008). Este aspecto en común viene reforzado por Silva (2010) cuando refiere la necesidad de toda una estructura que convalide la adquisición de esas competencias, en la que los entrenamientos sean configurados de manera que los jóvenes puedan manifestarlas, bajo el constante reforzamiento y una comunicación abierta y bidireccional. Larson (2000) concluye que el aprendizaje para el desarrollo positivo de jóvenes se da mejor en actividades estructuradas de participación voluntaria, como en el deporte y las artes, en donde hay la rara combinación de motivación intrínseca con alto nivel atencional, un ejemplo de estas actividades son los proyectos sociales que atraen a los jóvenes que buscan equipos deportivos.

Los entrenadores de proyectos sociales en Brasil actúan casi siempre solos en la labor educativa, y tienen recursos precarios para entrenar en barrios caracterizados por el alto grado de vulnerabilidad/ riesgo social. Asimismo, coordinan equipos a donde acuden voluntariamente decenas de deportistas que permanecen durante muchas temporadas. Así que el objetivo de este estudio es investigar cómo los entrenadores de proyectos sociales en Brasil enseñan competencias de vida a partir de sus filosofías, estilos de comunicación, rutinas y estrategias de trabajo empleadas en sus entrenamientos.

\section{Método}

\section{Participantes}

Se entrevistó un total de seis entrenadores de proyectos sociales (cinco hombres y una mujer). Cuatro de ellos (los entrenadores identificados como 2, 3, 4 y 5 ) entrenaban equipos de fútbol campo masculino, el entrenador 3 también dirigía un equipo femenino. El entrenador 1 trabajaba con futbol sala 
y baloncesto masculinos, y el entrenador 6 con voleibol mixto y fútbol sala femenino. Todos competían en las ligas de la ciudad de Porto Alegre, Brasil. Tenían entre 29 y 56 años $(M=39.1, \mathrm{DT}=9.4)$ y su experiencia como entrenadores era de dos hasta 32 años $(\mathrm{M}=12.3$, DT=11.8). En cuanto a su formación, cuatro entrenadores tenían algún postgrado universitario en educación física, otros dos estaban finalizando el grado en el mismo curso. Dos entrenadores trabajaban en un centro privado, tres en centros del ayuntamiento, mientras la entrenadora llevaba un proyecto de forma autónoma, sin ayudas de instituciones. Cada entrenador coordinaba por lo menos tres equipos de franjas de edad distintas, con jóvenes entre 7 y 18 años.

\section{Procedimiento}

En primer lugar se contactó con la Secretaria Municipal de Esporte, órgano del ayuntamiento que organiza la liga de la ciudad, para comprobar cómo funcionaban los proyectos deportivos vinculados a ella. En una cita con el principal responsable de la liga municipal, basándonos en el proceso empleado por Gilbert y Trudel (2004), se presentaron los objetivos del estudio y se pidió la colaboración de entrenadores con las siguientes características: a) que dirigieran el mismo equipo desde hacía más de una temporada y media, garantizando así la convivencia con los jóvenes durante toda una categoría; b) que sus equipos tuviesen buena reputación en lo que concierne al juego limpio y disciplina; c) que la participación en los equipos fuera voluntaria y gratuita; d) que sus equipos perteneciesen a barrios reconocidos por la baja renta y el riesgo de exclusión social.

Se realizó el contacto telefónico con los entrenadores confirmando las informaciones recibidas sobre los equipos, se programó una cita para una charla y, cuando fuera posible, observar un entrenamiento. Los objetivos del estudio fueron presentados a los entrenadores de forma presencial y todos aceptaron participar voluntariamente. Al inicio de la entrevista se garantizó el anonimato y la confidencialidad, además de la posibilidad de no contestar a cualquier pregunta. Con el consentimiento explicado y firmado, se pasó a la realización de las entrevistas, que tuvieron una duración entre 41 y 157 minutos $(\mathrm{M}=74 \mathrm{~min})$. El entrevistador fue el primer autor, experto en psicología deportiva, entrenador de fútbol titulado, con experiencia en entrevistas semi estructuradas del mismo contenido, realizadas bajo la supervisión del Grupo de Estudios de Psicología del Deporte de la Universitat Autònoma de Barcelona. Tras el análisis de resultados, y la confirmación de que se había logrado la saturación teórica descrita por Guest, Bunce y Johnson (2006), se consideró suficiente el número de entrevistas realizadas.

\section{Instrumento}

Para las entrevistas se utilizó el mismo guión de Marques et al. (2013), traducido al portugués y adaptado culturalmente por dos expertos bilingües. El guión ${ }^{1}$ contiene ocho bloques de preguntas abiertas, empezando con datos personales, de formación y experiencia deportiva, seguido de datos sobre los equipos que entrenaban en el momento de la entrevista. El tercer y cuarto bloque abordaban sus filosofías y estilos de entrenamiento, y el quinto preguntaba directamente sobre las competencias de vida que enseñaban y las estrategias utilizadas. El sexto bloque, que no existía en el estudio anterior, fue creado utilizando cuestiones adaptadas del guión de Camiré (2012) con preguntas sobre cómo y cuáles competencias se adquieren y pueden ser transferidas por los jóvenes. Posteriormente, se les pedía para hablar de las principales dificultades encontradas en sus entrenamientos, además de cómo trabajaban temas como la deportividad, el abuso de sustancias y el desarrollo de la autonomía en el equipo. Finalmente, se solicitaba una evaluación global de su trabajo como educador y entrenador, así como consejos y sugerencias para la mejora del tema en la ciudad.

\section{Análisis de datos}

El contenido de las entrevistas fue analizado por dos expertos, el primer autor y un psicólogo miem-

1 El guión se puede solicitar al primer autor por correo electrónico 
bro del grupo de investigación en psicología del deporte y competencias de vida, con experiencia en análisis cualitativo, que identificaron las meaning units (MU) existentes en cada una de ellas, según el modelo de análisis cualitativo para datos no estructurados de Côté, Salmela, Baria y Russell (1993). Una MU es un segmento de texto, frase, o parágrafo, que es entendible por sí mismo y contiene una idea, episodio o sección de información expresada por el entrevistado de manera clara y objetiva. Primero, ambos expertos seleccionaron las MU de cada entrevista discutiendo la relevancia de cada una de ellas hasta llegar a un consenso, además de nombrarlas con una etiqueta provisional.

Tras seleccionar $270 \mathrm{MU}$ entre todas las entrevistas, estas fueron agrupadas según sus etiquetas y reorganizadas en categorías por similitud de contenido. Así, fueron elegidas aquellas MU que representaban mejor cada categoría, teniendo en cuenta que algunas explicaban la filosofía de los entrenadores y otras, cómo las llevaban a cabo de forma práctica, es decir, las estrategias. Esas $74 \mathrm{MU}$ fueron distribuidas en 28 categorías que fueron discutidas y reajustadas en cuatro temas de orden superior etiquetados inductivamente: Trabajo en Equipo, Disciplina, Actuación y Vínculo, y Educación Integral. Finalmente, para garantizar la credibilidad del proceso, un tercer experto del grupo de investigación en psicología del deporte y competencias de vida, que no había participado de las etapas anteriores de la investigación, analizó una lista de todas las MU y aparte otra lista con los temas y sus categorías, eligiendo qué filosofías y estrategias estaban más relacionadas unas con otras. La discusión con los otros dos expertos condujo a la reorganización de algunas categorías, y a la elección de aquellas MU más representativas de cada categoría.

\section{Resultados y discusión}

Los resultados de la investigación sobre cómo piensan y actúan los entrenadores de equipos con respecto a la enseñanza de competencias de vida fueron clasificados en cuatro grandes temas: Trabajo en Equipo, Disciplina, Actuación y Vínculo, y Educación Integral, cada uno con sus categorías ${ }^{2}$ y estrategias.

\section{Trabajo en equipo}

Todos los entrenadores entrevistados subrayaron la importancia de fomentar el trabajo y el espíritu de equipo entre los jóvenes bajo su orientación. La cooperación fue frecuentemente mencionada como un valor que debe ser trabajado, como comenta el entrenador 1:

"Es tener un grupo muy heterogéneo y hacer que trabajen por la misma cosa, los mismos objetivos. Trabajar por el grupo, aunque haya poco en común. Lo que tienen en común cuando entran en la pista, es el fútbol".

Por lo tanto, es fundamental la generación de un clima positivo en el equipo para que haya buenas relaciones entre todos y apoyo en lugar de críticas:

“(...) es un botón que se presiona todos los días. Una buena relación de grupo en el deporte colectivo es una necesidad. No es posible trabajar, o hacer un deporte colectivo, con un grupo inmenso sin relacionarte bien con la gente. (E1)"

[Hago] ejercicios en que perciban la importancia del trabajo en grupo para el deporte, cuando hay que apoyar al colega que comete un error o animar a aquél que es novato. En lugar de regañar, yo digo: Hay que animar a la gente que está empezando, ustedes también empezaron alguna vez. (E6)

El cuidado con la inclusión de todos en las actividades del equipo fue destacado en el proceso de formación de un grupo unido:

Entreno para la competencia, todavía no me olvido del enfoque del proyecto social. Tengo que cuidarme mucho. Incluso para que los que no son buenos jugadores no se sientan perjudicados y no abandonen el equipo. Yo siempre tengo esa preocupación en acordarme de que es un proyecto social que incluye a todos. (E5)

2 Las categorias estan marcadas en negrita 
Una estrategia utilizada por el entrenador 2 es hacer que todos jueguen entre ellos, incluso los que no tienen lazos de amistad: "a veces les dejo elegir los equipos, eligen a sus amigos, y, antes de empezar, cambio a los capitanes".

Asimismo, con el tiempo, se acaba creando la amistad entre los jóvenes de los equipos, como en la declaración del entrenador 1 :

[El equipo] siempre está en contacto, se encuentra y juega en otros sitios con los chicos de escuelas privadas. No veo en que otro medio que no sea el deporte ellos podrían encontrarse. Están en barrios completamente distintos, opuestos en todos sentidos geográficamente y económicamente.

Algunos entrenadores incluso intentan dar las mismas oportunidades a todos para ejercitar lo que han aprendido en entrenamientos, o incluso en las competiciones:

En el partido hacer que todos los niños puedan probar situaciones de juego. Así que no solamente el mejor va tirar el córner, el saque de banda, el tiro libre. El adulto que está coordinando debe disolver eso en el grupo. (E2)

Todos van a jugar. Yo tenía un equipo titular, sin embargo, no he excluido a nadie por jugar mejor o peor. Si estuviera en un club, con enfoque de competición, pondría los mejores a jugar y los otros al banquillo. (E6)

Luego los entrenadores buscan y logran la mejora personal y colectiva en sus equipos, como bien explica el entrenador 6 :

Pues cuando aquel que no es tan bueno mejora, todo el grupo mejora. Les hago que el nivel del juego mejore y quien es mejor quiere jugar con más nivel. (...) Busco coger los más experimentados que tienen más técnica, más tiempo en el equipo y emparejarles con alguien. "Hoy vas hacer el calentamiento con ese que está empezando, ayúdalo con el toque, explícale”. Y va bien.

También en el trabajo en equipo, es estimulada la responsabilidad con el grupo como un todo como mencionaron los entrenadores 4 y 2 :
Hay situaciones de juego, por ejemplo, que uno hace el pase mal porque está perezoso, y el otro equipo va por ahí y hace un gol. Doy este ejemplo para que todo lo que hacemos tenga que ser bien hecho. Si uno hace algo mal hecho, va perjudicar a otra persona, su grupo será castigado.

Ellos ayudan a llevar las cosas a la cancha. Todo es un proceso en el que hay que dar su contribución al equipo. No puede ser que uno llegue aquí y se ponga las botas, pantalones, calcetines, a jugar y listo. Hay que tener algo más, el vínculo, la trayectoria.

La investigación de Marques et al. (2013) demostró que una categoría de competencias de vida trabajada por entrenadores de proyectos sociales en España es la relación, que incluye: cooperación, compañerismo y la integración (cultural en el caso del contexto europeo), lo que parece estar en consonancia con la práctica de los educadores brasileños entrevistados respecto a las categorías Cooperación, Inclusión y Amistad. El dar las mismas oportunidades también es una estrategia para la enseñanza de competencias de vida en el referido estudio. El establecimiento de un clima motivacional positivo por parte del entrenador es una de las pautas del MAC (Smith et al., 2007) programa de intervención para entrenadores que enseña directrices de actuación en la búsqueda de beneficios asociados a un clima orientado hacia la maestría.

Por otra parte, la Teoría de la Autodeterminación (Self-Determination Theory; Deci \& Ryan, 2000) está fundamentada en la creencia de que una de las bases de una motivación autodeterminada es la satisfacción de tres necesidades básicas innatas (i.e., autonomía, competencia y relación). Esta teoría nos ayuda a comprender por qué fomentar las relaciones dentro del grupo es tan importante para mantener a los jóvenes motivados en participar del equipo. Lo mismo sucede con la necesidad básica de competencia, que tiene que ver con las posibilidades de aprendizaje y perfeccionamiento que alguien percibe que tiene sobre alguna habilidad, y explica la categoría de Mejora Personal y Colectiva. 


\section{Disciplina}

Todos los entrenadores se preocupan por la disciplina de sus equipos en todos sus aspectos. Primeramente exigen el respeto de sus deportistas, Entre ellos mismos, a los adversarios, al árbitro y a las reglas, conjuntamente con el juego limpio, como bien ejemplifica el entrenador 6 :

Yo trabajo mucho con ellos la honestidad en el juego, digo "Viste que ha salido el balón, acusa, tocó la mano, acusa". El objetivo no es ganar a cualquier coste, ayuda y respeta al árbitro y a tus compañeros. (...) Porque creo que todo el mundo que forma parte de un grupo, un equipo deportivo, en algún momento aprende a tratar con diferentes personas de una mejor manera, porque dentro del grupo hay múltiples personalidades. Y uno aprende a manejar las diferencias y a respetar a los demás. Esto es lo que uno lleva para la vida, para el hogar y para la escuela con sus colegas.

Además, hay la preocupación con el saber perder y ganar:

Desde el ganador saludar al perdedor, es una cosa de honor, un deporte de caballeros, ética, eso tiene que ocurrir. Así que el ganador saluda por el honor de haber tenido la oportunidad de haber jugado con usted. (E2)

Los entrevistados en un primer momento tienen establecido un reglamento para las conductas permitidas o no permitidas en entrenamientos y partidos, y utilizan distintas estrategias cuando notan que los ánimos se calientan, como la entrenadora 5:

A veces hacemos un ejercicio, cuando hay mucha querella yo detengo "iPara! iQue cada uno cuente hasta diez! "En medio del partido, me da igual si está bueno. Pues primero está la armonía, equipo que pelea entre sí nunca va ganar a nadie.

O cuando dos jóvenes discuten: "Saco, y no hablo. Lo que quieren es platicar, sácale, aléjale y seguimos aquí. Sólo después es que hablo con ellos por separado. (E4)". Lo mismo pasa en partidos, como relata el entrenador 3: "Lo saco, no pasa nada si pierdo el partido. Pero no va a ser expulsado, no habrá pelea porque nunca sucedió".

Todos relataron tener como "regla de oro" que "quien se pelea sale del equipo" idea resumida por el Entrenador 2: No se puede reaccionar con violencia. Hay que tener un pacto de no violencia. Tal vez es la primera regla básica, no podemos hacer daño a nadie aquí. Ni desde el punto de vista moral, ni tampoco desde un punto de vista físico.

Así que hay punición a quien se pelea, aunque con la posibilidad de perdón según en qué caso:

Por supuesto que hoy ha vuelto al equipo [un chico que fuera sacado por pelear], mucho mejor desde su regreso, sufrió en la piel lo que es ser excluido del grupo, hay que trabajar para volver.

Pues como justifica la entrenadora 5: "No, de nada vale [expulsar] aquél niño [el problemático] es el que más me interesa a mí, es una forma de mantenerlo cercano a mí."

El respeto a todos los participantes de la escena deportiva y la deportividad son tópicos comunes en la actuación de los entrenadores preocupados por la enseñanza de competencias para la vida. Los mejores entrenadores del estudio de Gould et al. (2007) también responsabilizan a los jóvenes de sus actos, además de castigar las infracciones que ocurren. Los entrenadores entrevistados por Camiré et al. (2012) crean y utilizan expresiones para hacer recordar a los alumnos que deben portarse bien, pues representan a su escuela y barrio. Lo mismo pasó con los participantes del estudio de Marques et al. (2013) que buscaban trabajar una serie de competencias sobre la educación, y solamente hacían críticas en sus entrenamientos al observar malas conductas.

\section{Actuación y vínculo}

Los entrenadores utilizan distintas estrategias sobre cómo actuar en el trato personal con los jóvenes de sus equipos con el objetivo de crear un buen vínculo. Estimular el esfuerzo y reforzar positivamente son las prácticas más comunes, a nivel personal y 
grupal: "Hablar, animar, estimular el esfuerzo, retar y ofrecer situaciones para que el niño tenga que pensar, plantear problemas que tengan que solucionar dentro de las situaciones de partido." (E2). "Podemos perder, como hemos perdido muchas veces, pero tenemos que tener más ganas que los otros." (E4). "Yo apoyo mucho, enalteciendo y valorando su crecimiento al darme cuenta de su dedicación, su compromiso y su aprecio en los entrenamientos y partidos." (E2).

Incentivar la autonomía y dar feedback también son prácticas comunes: "Quién dicta las reglas soy yo, pero respetando sus opiniones y tratando de mejorar lo que pensamos que está mal, teniendo feedback de ellos también." (E4). "Darles la oportunidad de elegir a sus pares en distintos juegos. En un juego, una carrera de relevos, un juego cooperativo, como la elección del propio equipo."(E2). "Siempre, antes de cada entrenamiento nos encontramos con ellos y pasamos algunas pautas, además de reflexionar sobre cada práctica." (E4).

Otro punto importante para la creación y mantenimiento del vínculo es el convivir y charlar con los chicos y chicas de sus equipos no solamente en los entrenamientos y sobre el deporte, como nos comentaron los entrenadores 2 y 1 respectivamente:

"[De la comida colectiva que hay después del juego] no debe ser algo sólo para satisfacer el hambre, y mientras tanto, hablar de alguna jugada, o ahora nos toca jugar contra este equipo. Es más un espacio de socialización”.Hay, por supuesto, un vínculo afectivo muy fuerte con los chicos. Hay unos pocos que están tres, cuatro, cinco años allí. Todo el año entrenando y saliendo a partidos todas las semanas. [...] Con el tiempo empezamos a crear otra relación, como de amistad. Hablamos de otras cosas, no solo de baloncesto. Vamos a hablar de la escuela, a veces de las novias, o que no está bien por algo, o incluso si hay algunos dramas familiares.

La enseñanza con diversión y afecto, además de ser un buen ejemplo, es igualmente una estrategia que ayuda en las relaciones con los jóvenes, como se puede apreciar "[Me aburro de entrenar] no tiene sentido, nadie está obligado a venir. No es profesional, hay que venir con goce, divertirse y jugar con sus compañeros, con tus amigos, pero sin lío." (E6).
[...] Veo que la mayoría han sufrido maltratos en su hogar y ya están acostumbrados. Vinieron de otros equipos en los que el entrenador chillaba y regañaba. Les pegan en casa y pelean en la calle. Así que reflexioné y pensé: "Voy a hacer todo diferente con ellos solamente basado en el amor y el afecto. Incluso los más molestos, esos son los más necesitados de afecto. Veo que va bien. (E5)

Los veo allí en la cancha como si fueran un montón de palillos de fósforo, algunos pueden explotar en cualquier momento. Así que si me pongo a gritar es como estar echando alcohol para que ocurra una explosión general. Intento ser lo más tranquila posible, como son mis modales también, siempre tranquila para así mantener al equipo. Creo que es por eso que nunca hubo peleas en el equipo, nunca se pelearon en el campo. (E5)

Es importante subrayar el hecho de que solamente el entrenador 2 adopta capitanes para la elección de equipos en los entrenamientos y valora esa función de liderazgo, lo que no es práctica común en los otros equipos entrevistados en Brasil, y todavía ocurre con todos los entrenadores participantes de los estudios referidos que se efectuaron en España, Canadá y EEUU.

La relación entrenador-deportista, a partir de su estilo de comunicación, el cuidado, el trato, la convivencia y el ser un buen modelo es una de las bases para el trabajo de los entrenadores que aparece en diversos estudios que exponemos en esta investigación (Camiré et al., 2012; Falcão et al., 2012; Gould et al., 2007). El modelo de Gould y Carson (2008) ofrece un entendimiento más amplio, y acaba por contemplar los resultados de esas investigaciones. En lo que denomina "Estrategias directas de entrenamiento" habla de la cantidad y cualidad del refuerzo e instrucciones, de promover oportunidades de liderazgo y de la toma de decisiones por parte de los jóvenes y para darles atención individualizada. Partiendo de la teoría de la autodeterminación, el apoyo a la autonomía por parte del entrenador (Deci et al., 2000) apunta a que un instructor que ofrezca un ambiente donde el practicante se perciba con voz, posibilidad de elección y control, estará facilitan- 
do la motivación intrínseca y la pasión del joven por el deporte.

\section{Educación Integral}

Los entrenadores entrevistados compartían en sus filosofías la preocupación de la enseñanza de competencias de vida a través del deporte, y durante el aprendizaje del mismo. Todos relataron que el deporte es un instrumento, y no el objetivo de su trabajo en los proyectos de los que forman parte. Los entrenadores 4 y 2 explican esa idea "Reunir a los chicos y ofrecerles una escuela, un trabajo, una formación, una educación fuera de la escuela. El enfoque de las clases no es competitivo.".

Yo diría que es una filosofía basada en la educación a través del deporte, la educación de este deporte, pero siempre teniendo una mirada más grande de sus vidas. Un proceso de la educación para la vida.

Así [...] Busco ser profesor y educador antes que ser entrenador.

Por eso todos se preocupan en la educación del civismo y de incentivar el estudio y el trabajo de los chicos y chicas con que conviven.

Hay muchos, que llegaron aquí y que parecían una bestia, y ahora saben ir a un partido, entrar en una cancha. Saben hacer su juego, y al terminar, subir al autobús, ir a casa tranquilo. Cosa que muchos no sabían. No existe en sus casas. (E1)

"Con el paso del tiempo y con la imposición, enseñando algunos aspectos, hoy tenemos a chicos que trabajan en puestos de trabajo que les recomendamos nosotros". (E3)

Incluso manteniendo contacto con sus padres para el seguimiento escolar, reportar malas conductas o influencias, o incluso tratar de problemas grandes como el uso de drogas y abuso de sustancias "Muchas madres me buscan por la escuela. Creen que si hablo con el chico, él va ir bien en el colegio. $\mathrm{O}$ que amenace al chico que no va a entrenar más." (E5).
Finalmente, la enseñanza deportiva, como práctica que queda para la vida actual y futura, también forma parte de la labor educativa de los entrenadores participantes: "El deporte, el ocio, o la recreación, quedan en la vida de las personas, como un medio de educar provee calidad a la vida. A quien le guste hacer deporte, independientemente de lo que haces para ganarte la vida, aquellos que aman el deporte y tienen oportunidad de practicar, practicarán." (E1).

La utilización del deporte como herramienta para transformar la vida de estos jóvenes y transmitir competencias que quedarán y serán útiles en sus escuelas, trabajos, familias, etc. es el aliciente y la principal función de todos los entrenadores participantes de esta investigación, así como de los estudios de Camiré et al. (2012), Gould et al. (2007) y, Marques et al. (2013), sobre el tema. El incentivo a los estudios y trabajo, la enseñanza de valores y competencias positivas en un ambiente donde los chicos y chicas estén motivados a participar y hacer caso al adulto que gestiona es considerado más importante que ganar partidos y copas, como destacó Vella et al. (2011). Eso puede ser considerado el gran rasgo de quien se dedica a llevar un proyecto social y no un club, lo que diferencia al entrenador-educador social, del entrenador de un club profesional.

\section{Conclusiones}

Los resultados indican que los entrenadores entrevistados tienen un perfil análogo al modelo de entrenamiento de competencias de vida de Gould et al. (2007) pues tienen: (a) filosofías fundamentadas; (b) vínculo y buena relación con los deportistas; (c) consideración del entorno donde trabajan; y (d) estrategias para la enseñanza de competencias de vida. En comparación con el estudio de Marques et al. (2013) los participantes brasileños tienen mejor formación universitaria y más experiencia, aunque tienen las mismas dificultades para describir estrategias específicas para la educación de las competencias.

Como los entrenadores españoles, ellos poseen buena voluntad y ganas de realizar la labor educativa, aunque carecen de planteamiento y sistemática 
para la ejecución como ocurre con el entrenamiento táctico, técnico y físico en el deporte. En esta situación, Danish, Forneris y Wallace (2005) proponen que se trabaje una competencia cada vez, entrenando y hablando sobre su concepto con ejemplos de cómo se aplica en situaciones no deportivas. También sugieren que se ofrezcan oportunidades para que los jóvenes empleen las competencias elegidas, reflexionen e intercambien esa experiencia, compartiendo con sus compañeros los éxitos y fracasos en el intento y reforzando su aprendizaje.

Una limitación importante para que eso ocurra es la gran cantidad de jóvenes que cada entrenador tiene bajo su responsabilidad, además de la poca ayuda interna (nadie poseía un segundo entrenador) y externa, y el hecho de que todos tenían otras ocupaciones. Tampoco existe un espacio de intercambio de experiencias entre estos entrenadores que actúan en realidades similares y afrontan las mismas dificultades como la pobreza, el tráfico de drogas, y la falta de apoyo para el mantenimiento de las actividades de los equipos.

Con la continuación de nuestras investigaciones deseamos proponer un programa de formación a estos entrenadores, inspirado en los que ya demostraron resultados eficaces, como el MAC, SUPER y PAPE, que sea coherente con la realidad cultural y económica de los países de América Latina. Profundizar en estudios de cómo los jóvenes adquieren y transfieren las competencias para sus vidas es otro desafío para futuras investigaciones. Finalmente, es importante mencionar la limitación de este estudio con un número reducido de participantes, lo que imposibilita la generalización de nuestros resultados.

\section{Referencias}

Camiré, M. (2012). Facilitating Positive Youth Development through High School Sport. (Doctoral disertation, University of Ottawa, Canada). Retrieved from https://www.ruor.uottawa.ca/handle/10393/22903

Camiré, M., Trudel, P., \& Forneris, T. (2012). Coaching and transferring life skills: Philosophies and strategies used by model high school coaches. The Sport Psychologist, 26 (3), 243-260.
Côté, J., Salmela, J. , Baria, A., y Russel, S. (1993). Organizing and interpreting unstructured qualitative data. The Sport Psychologist, 7(2), 127-137.

Côté, J., Salmela, J., Trudel, P., Baria, A., \& Russell, S. (1995). The coaching model: A grounded assessment of expert gymnastic coaches' knowledge. Journal of Sport Exercise Psychology, 17 (1), 1-17.

Danish, S., Forneris, T., \& Wallace, I. (2005). SportBased Life Skills Programming in the Schools. Journal of Applied School Psychology, 2 (21), 41-62.

Danish, S. \& Nellen, V., (1997). New roles for sport psychologists: Teaching life skills through sport to at-risk youth. Quest, 49 (1), 100-113.

Danish, S., Nellen, V., \& Owens, S. (1996). Teaching life skills through sport: Community-based programs for adolescents. En J.L. Van Raalte \& B. Brewer (Eds.). Exploring sport and exercise psychology (pp. 205-225). Washington, DC: American Psychological Association.

Deci, E., \& Ryan, R. (2000). The "what" and "why" of goal pursuits: Human needs and the self-determination of behavior. Psychological Inquiry, 11 (4), 227-268.

Falcão, W., Bloom, G., \& Gilbert, W. (2012). Coaches' perceptions of a coach training program designed to promote youth developmental outcomes. Journal of Applied Sport Psychology, 4 (24), 429-444.

Gilbert, W., \& Trudel, P. (2004). Role of the coach: How model youth team sport coaches frame their roles. The Sport Psychologist, 18 (1), 21-43.

Gould, D., \& Carson, S. (2008). Life skills development through sport: Current status and future directions. International Review of Sport and Exercise Psychology, 1 (1), 58-78.

Gould, D., Chung, Y., Smith, P., \& White, J. (2006). Future directions in coaching life skills: understanding high school coaches' views and needs'. Athletic Insight On-line, 3 (8), 28-38.

Gould, D., Collins, K., Lauer, L., \& Chung, Y. (2007). Coaching life skills through football: A study of award winning high school coaches'. Journal of Applied Sport Psychology, 1 (19), 16- 37.

Guest, G., Bunce, A., \& Johnson, L. (2006). How many interviews are enough? An experiment with data saturation and validity. Field Methods, 18, 59-82. 
Holt, N., \& Neely, K. (2011). Positive youth development through sport: a review. Revista de Iberoamericana de Psicología del Ejercicio y el Deporte, 2 (6), 299-316.

Larson, R. (2000). Toward a psychology of positive youth development. American Psychologist, 55 (1), 170-183.

Marques, M., Nonohay, R., Koller, K., Gauer, G., \& Cruz J. (2015) El estilo de comunicación del entrenador y el clima motivacional percibido en sus equipos. Cuadernos de Psicología del Deporte, 15 (2) 47-54.

Marques, M., Sousa, C., \& Cruz, J. (2013). Estrategias para la enseñanza de competencia de vida a través del deporte en jóvenes en riesgo de exclusión social. Apunts Educación Física y Deportes, 112, 63-71.

Silva, P. (2010). Ensino e transmissão de competências de vida por via da prática desportiva (Tesis de maes- tria no publicada) Universidade do Porto, Porto, Portugal.

Smith, R., Smoll, F., \& Cumming, S. (2007). Effects of a motivational climate intervention for coaches on changes in young athletes' achievement goal orientations. Journal of Clinical Sport Psychology, 1 (1), 23-46.

Sousa, C., Cruz, J., Torregrosa, M., Vilches, D. \& Viladrich, C. (2006). Evaluación conductual y programa de asesoramiento personalizado (PAPE) a entrenadores de deportistas jóvenes. Revista de Psicología del Deporte, 15(2), 263-278.

Vella, S., Oades, L., \& Crowe, T. (2011). The central role of the coach in facilitating Positive Youth Development: Moving from theory to practice. Journal of Applied Sport Psychology, 23, 33-48. 
